\title{
EVALUATE THE PERFORMANCE OF AN SIGN LANGUAGE TRANSLATOR MOBILE APPLICATION
}

P. PERINDHA VALLI ${ }^{\# 1}$, K. CHANDINI $^{\# 2}, \quad$ D. LAABITHA
G. DURGA BHAVANI SHANKAR
$\# 4$, J. SANTOSHI KUMARI
$\# 1,2,3,4$ B.Tech Scholar, Department of Computer Science and Engineering,
Nadimpalli Satyanarayana Raju Institute of Technology (NSRIT), Sontyam, Pendurthi-
Anandapuram Highway, Visakhapatnam, India-531173.
Assistant Professor, Department of Computer Science and Engineering,
Nadimpalli Satyanarayana Raju Institute of Technology (NSRIT), Sontyam, Pendurthi-
Anandapuram Highway, Visakhapatnam, India-531173.

In current days mobile applications act as one of the major medium to access any task or events from one place to other place of the world. Android is a mobile operating system developed with the help of updated version of the kernel. This is mainly developed for touchscreen applications using devices like handheld tabs, smart mobiles and wireless devices. This current thesis aims at building a machine learning (ML) model that will be ready to classify the varied hand gestures used for fingerspelling in sign language. During this user-independent model, classification machine learning algorithms are trained to employ a set of image data and testing is completed on a totally different set of knowledge. In this proposed thesis we try to use a very clear and depth images for obtaining accurate result than compared to previous methods in the literature. Various machine learning algorithms are applied on the datasets, including Convolutional Neural Network (CNN). An effort is formed to extend the accuracy of the CNN model by pre-training it on the Imagenet dataset. However, a little dataset was used for pre-training, which gave an accuracy of $15 \%$ during training. Signing consists of fingerspelling, which spells out words character by character, and word level association which involves hand gestures that convey the word sense. Fingerspelling may be a vital tool in signing because it enables the communication of names, addresses and other words that don't carry meaning in the word-level association. In spite of this, fingerspelling isn't widely used because it is challenging to know and difficult to use. Moreover, there's no universal signing and really few people realize it, which makes it an inadequate alternative for communication.

Key Words: Android, Kernel, Machine Learning (ML), Convolutional Neural Network, Fingerspelling, Sign Language.

\section{INTRODUCTION}

As we know that disabled people face a lot of problems while handling novel technologies like: the use of a laptop, retrieving, reading, writing, printing and editing a text and so on. Reading a document is one of the complex tasks for the disabled people compared with normal person [1]. In current days, there is abundant progress for providing opportunities to bring a better quality of life to those who endure handicap and disabilities [2]. The main target for this current thesis is to design a novel language like Sign language [3], to address all the problems which we discussed so far. A sign language is a novel language which takes input manually rather than sound ways to convey the information. This sign language is formed mainly by gathering heterogeneous hand shapes with distinct 
orientations, movements and facial expressions. The sign language is formed mainly with its own sentence constructions using different shapes and symbols. The Portuguese sign language (LGP) is one of the languages which have a very low diffusion level in the deaf and hearing communities. For an effective communication, the deaf person needs to have the knowledge of LGP language in two sides: One in listening side and other at speaking side. Due to the lack of availability of the sign language, it is primary step for us to improve the access of sign language by developing some advanced applications which can replace the manual efforts[4].

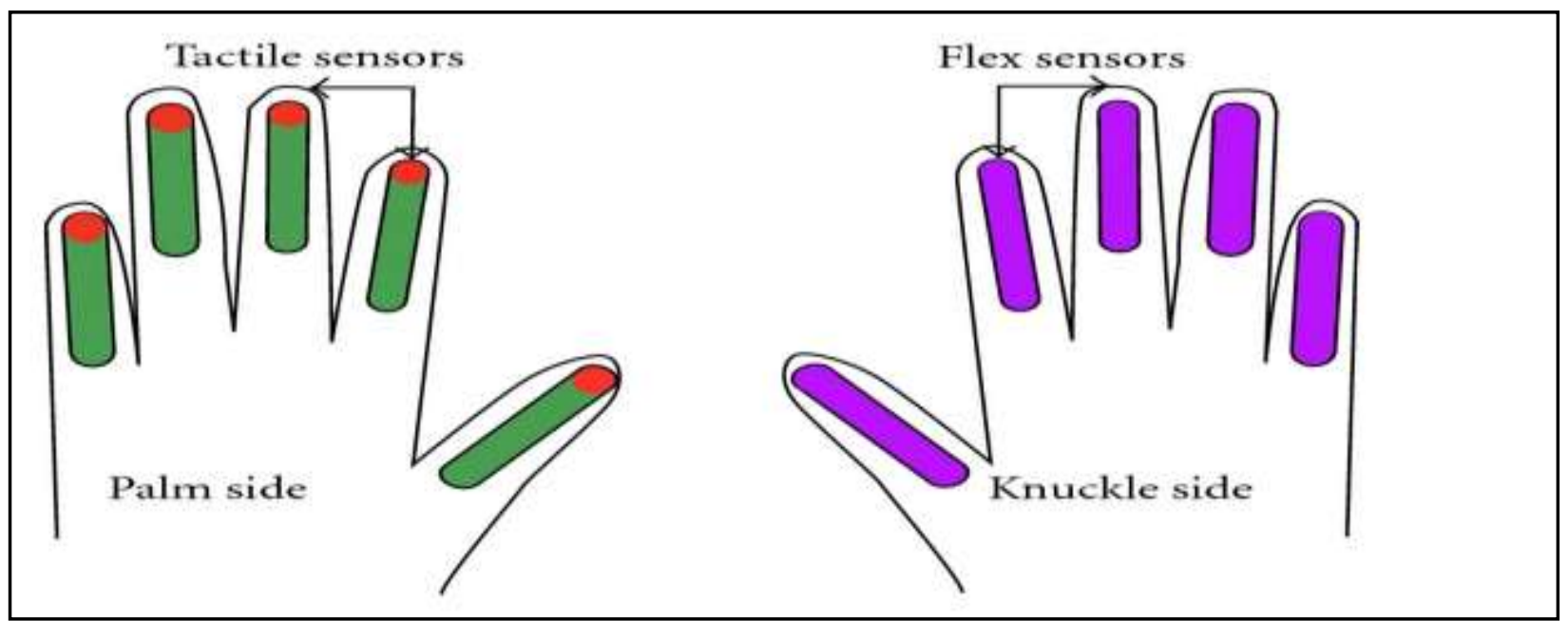

Figure 1. Denotes the Exploration of Sensor Gloves in Sign Language

From the figure 1, we can able to identify sign language is having two types of exploration: One is palm side and other is Knuckle Side. These two are having some sensor gloves to differentiate the task from a palm. If we see in the above figure 1, the palm side fingers are known as tactile sensors and all the five fingers in the palm side are identified as individual tactile with distinct meanings. If we see on knuckle side of the hand, they are termed as flex sensors.

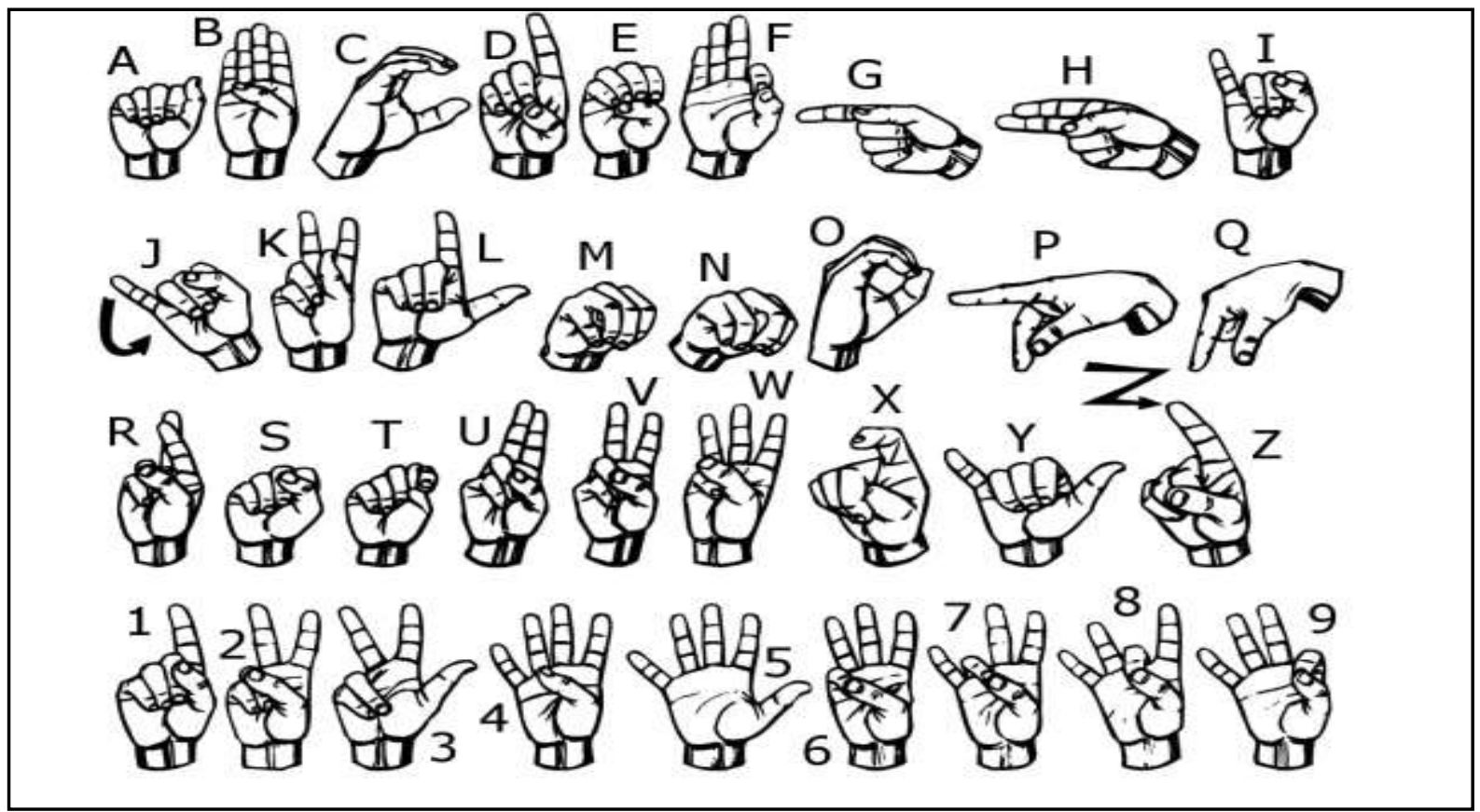

Figure 2. Denotes the Sample Glove that can be used to Translate Sign Language into Text and Speech 
From the above figure 2, we can clearly see a glove is shown in several ways and each and every picture is represented with unique identification.Here we can see totally 35 gloves, where the first 26 gloves are marked with alphabets $\mathrm{A}$ to $\mathrm{Z}$ and remaining 9 gloves are meant to map the numbers from 1 to 9 .Here these gloves are collective formed by collaborating palm and knuckle side sensors. These images are taken as test images and placed in the database for matching in training phase. The above construction is also studied by the language like Phonology, which refers to the study of some unique sounds present in human speech. In general the phonology tries to see different signs which are present in the conversation row with distinct hand signs.

This will be finding the meaning based on some parameters like:

- Configuration Parameter: This is nothing but finding the hand shape when it is doing the sign.

- Hand Orientation Parameter: This will be finding the palm pointing to.

- Hand Position Parameter: This will be finding how the final sign is finished.

- Motion Parameter: This will be finding the different types of movements which are performed by the hand while showing the sign.

For Example: Straight, swaying, circular.

- Hand Contact Point Parameter: This will be finding which part of the body the hand touched.

- Plane Parameter: This will be finding based on the distance of sign to the body.

- Non-manual Components Parameter: These are the additional information provided by the body like body leans front, it expresses future tense.

\section{BACKGROUND LITERATURE WORK}

In this section we mainly discuss about the background literature work that was carried out in finding the work that is related to sign language translator.

\section{MOTIVATION}

Sign language is a novel language through which communication is done without using any form of vocal sounds. They mainly 1 depend on the sign patterns like body language, arm orientation, hand movements, arm position and so on to figure out the intention of the blind people. This sign language is mostly used by deaf and disabled persons to communicate one with other. In current days almost more than 200 sign languages are in use and all are having common similarities based on sign symbols. In a recent survey it is found that nearly fifteen million people are having hearing impaired problems in India, making it the country with the largest number of deaf and largest number of sign language users. In order to overcome all these problems, we aim to construct and develop a novel sign language translator application. This application is created by taking a sample data set with more than 4000 characters under 24 distinct classes which can be formed with all 26 english letters, words and sentences in Indian Sign Language (ISL). In this current application we try to develop the text to sign language translator successfully and in the next phase we try to develop the sign to text conversion.

\section{DATA PREPROCESSING}

Generally this stage is very important in our current application as the data preprocessing plays a very vital role in the current application. In this section we mainly discuss about the methods that are used in the current applications. The various types of applications are explained in detail by the following figure 3.Data preprocessing is considered as the one of the most critical steps in a data mining process which mainly deals 
with the preparation of initial data set followed by transformation of the initial dataset. Data preprocessing methods are divided into following four categories as described below:

1. Data Cleaning method

2. Data Integration method

3. Data Transformation method

4. Data Reduction method

Of all the above four categories each and every category has its individual advantages and usage.Initailly the raw data what we take as input is entered into data cleaning method and once that category processes the data, the data will be cleaned and displayed with no errors. Now the data which is processed is now entered for transformation method, where a set of data is integrated based on any common attribute. Once the data is transformed now it will be entered into the data reduction category which is the final category. In this category the data will be represented in the form of tabular way with all the corresponding data into the table cells.

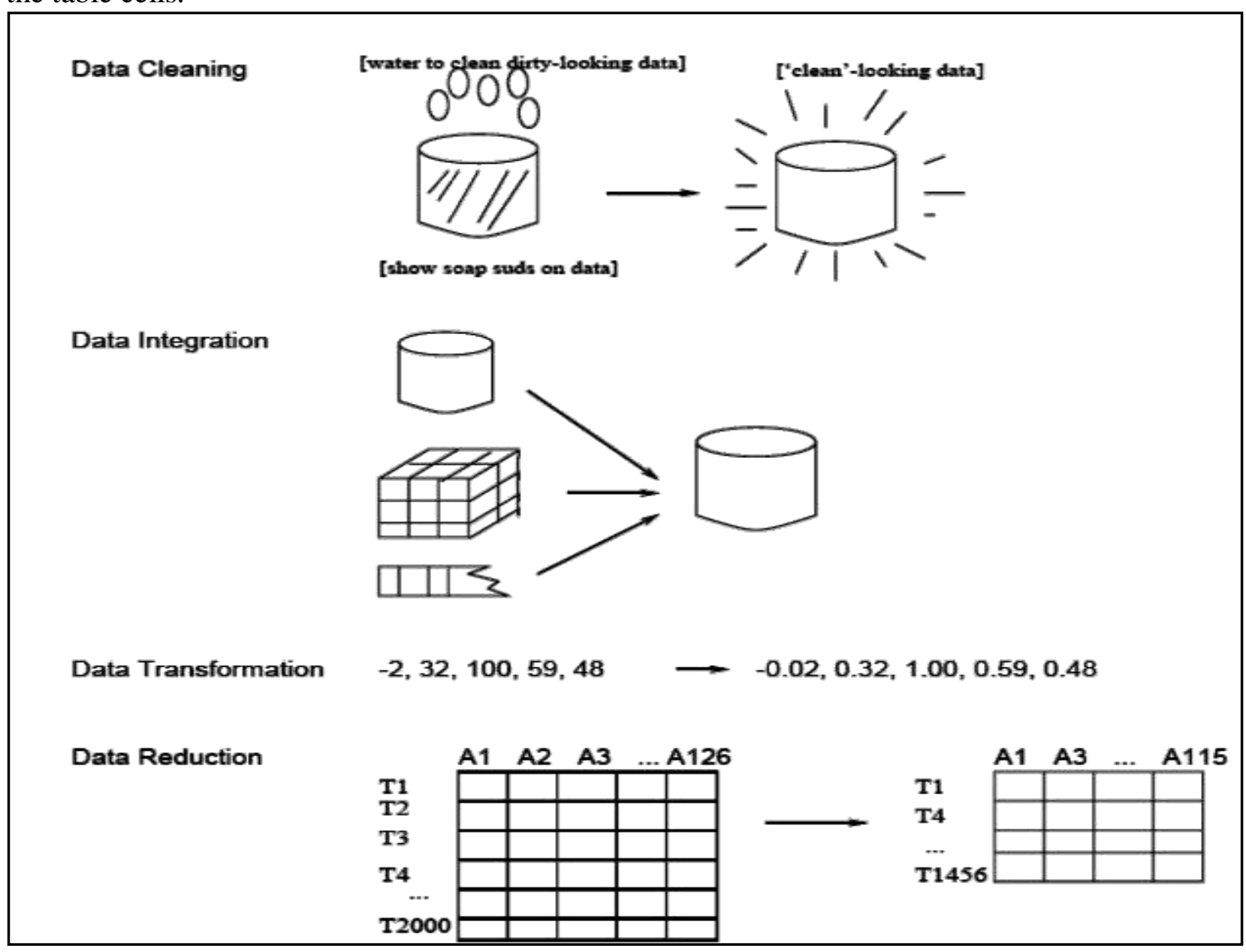

Figure 3. Denotes the Layered Approach of Data Pre-Processing Technique

From the above figure 3, we can clearly identify that data pre-processing has nearly 4 stages and in the current application we try to take several hand signs and form a data set. During the data set formation some of the images may be half formed, corrupted or not clear manner. So this data cleaning process will filter out such in complete images and try to form a data set containing clear and useful pictures. 
DOI: https://dx.doi.org/10.26808//rs.ca.i10v2.04

International Journal of Computer Application (2250-1797)

Issue 10 Volume 2, March-April 2020

\section{THE PROPOSED SIGN LANGUAGE TRANSLATION CLASSIFICATION USING MACHNE LEARNING TECHNIQUES}

$\mathrm{n}$ this section we mainly discuss about the proposed Sign Language Translator Classification technique using ML Approach.Now let us discuss about this proposed SEMSDAE Algorithm in detail as follows:

\section{PRELIMINARY KNOWLEDGE}

Initially we need to understand the basic functionality of Neural Network. In the branch of machine learning, ANN (I.e. Artificial Neural Networks) plays an important role in performing classification technique well compared with several other classification techniques. The ANN is mainly used in classification of several tasks like image, audio, words. This will come in different flavors based on the type of input data, for example if we want to classify the words, we try to use Recurrent Neural Networks (RNN) and for image classification we use Convolution Neural Network. In this section, we are going to build basic building block for $\mathrm{CNN}$ as we are using images as input data set for classifying various types of sign images.

In general the regular Neural Network contains 3 main layers like:

\section{Input Layers:}

This is the first layer in which we try to divide the input into number of neurons. Here the neurons are nothing but equal to pixels of an image.

\section{Hidden Layer:}

This is the second layer in the CNN, where the input from the first layer is send to this hidden layer. There may be many hidden layers present in this model depending upon our model and data size. Each hidden layers can have different numbers of neurons which are generally greater than the number of features. The output from each layer is computed by matrix multiplication of output of the previous layer with learnable weights of that layer and then by addition of learnable biases followed by activation function which makes the network nonlinear.

3. Output Layer: In this layer the output from the previous hidden layer as given as input to this layer and then they are constructed into a logistic function like sigmoid which converts the output of each class into probability score of each class.

The final resultant output is then obtained from the previous stages and they are forwarded to a final step called as feed forward error using an error function like cross entropy, square loss error etc. Finally we will calculate the derivatives called as back propagation which is used to minimize the loss occurred in these processing.

\section{LAYERS USED TO BUILD CONVNETS:}

A covnets is a sequence of layers, where each and every layer tries to convert one volume to another through differentiable function.

Let's take an example by running a covnets on of image of dimension $32 \times 32 \times 3$.

A) Input Layer: This layer holds the raw input of image with width 32, height 32 and depth 3.

B) Convolution Layer: This layer computes the output volume by computing dot product between all filters and image patch. Suppose we use total 12 filters for this layer we'll get output volume of dimension $32 \times 32 \times 12$.

C) Activation Function Layer: This layer will apply element wise activation function to the output of convolution layer. Some common activation functions are $\operatorname{RELU:~} \max (0, \mathrm{x})$, Sigmoid: $1 /\left(1+\mathrm{e}^{\wedge}-\mathrm{x}\right)$, Tanh, Leaky RELU, etc. The volume remains unchanged hence output volume will have dimension 32 × 32 × 12 . 
D) Pool Layer: This layer is periodically inserted in the covnets and its main function is to reduce the size of volume which makes the computation fast reduces memory and also prevents from over fitting. Two common types of pooling layers are max pooling and average pooling. If we use a max pool with $2 \times 2$ filters and stride 2 , the resultant volume will be of dimension $16 \times 16 \times 12$.

\section{VGG -16 ARCHITECTURE ( VISUAL GEOMETRY GROUP) FOR THE DETECTION OF SIGN LANGUAGE}

VGG-16 is a convolutional neural network architecture, it is named as VGG-16 because it contains 16 layers. The layers present in the VGG-16 are as follows: connected layers.

Convolutional layers, Max Pooling layers, Activation layers, Fully

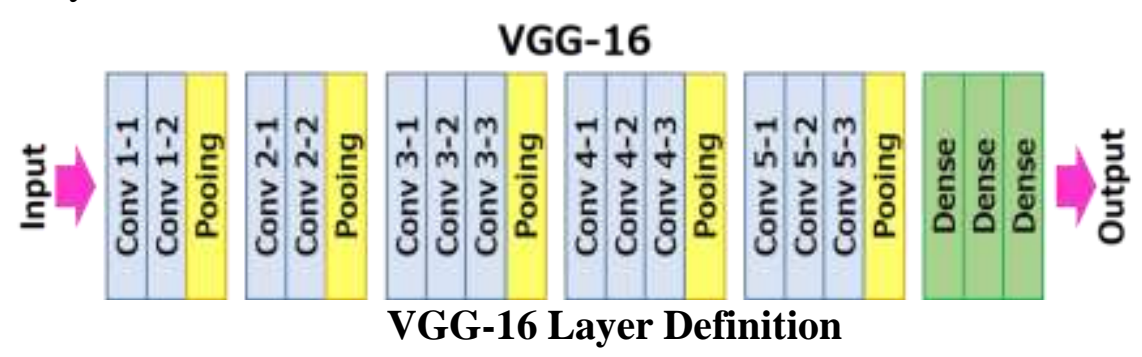

There are 13 convolutional layers, 5 Max Pooling layers and 3 Dense layers which sums up to 21 layers but only 16 weight layers. Conv 1 has number of filters as 64 while Conv 2 has 128 filters, Conv 3 has 256 filters while Conv 4 and Conv 5 has 512 filters.VGG-16 network is trained on ImageNet dataset which has over 14 million images and 1000 classes, and acheives $92.7 \%$ top-5 accuracy. It surpasses AlexNet network by replacing large filters of size 11 and 5 in the first and second convolution layers with small size 3x3 filters.Now, we will train an image dataset with VGG-16 in Keras. So, let's get started hands-on.

\section{PROPOSED ARCHITECTURE}

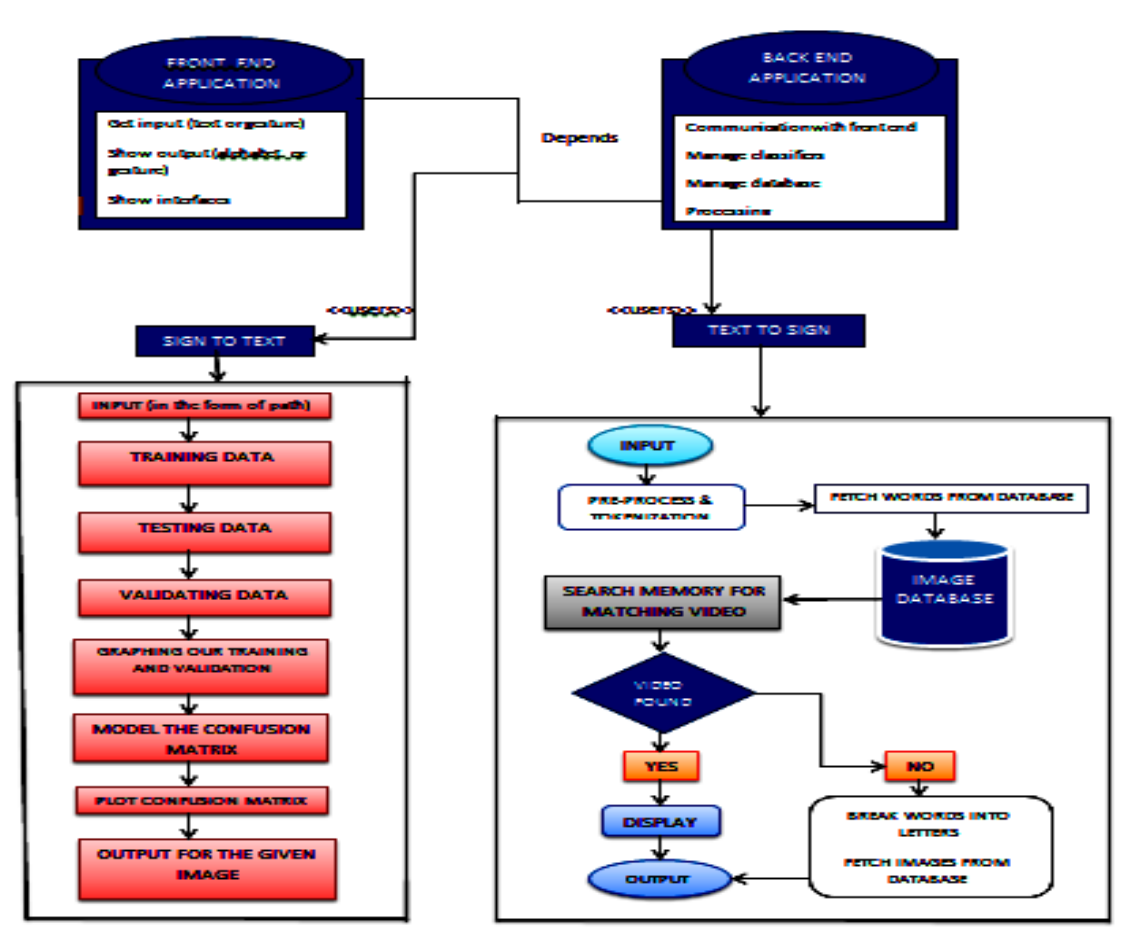




\section{SYSTEM IMPLEMENTATION}

Implementation is the stage where theoretical designs are converted into programmatically manner. In this application we mainly divide the application into 3 main modules for doing text to sign language translator. Now let us discuss about each and every module in detail. They are as follows:

\section{1) Load the Dataset}

In this module we try to create a dataset containing 40 characters containing alphabet characters, numbers and some messages which is in the same folder.

\section{2) Pre-Processing of Data Set}

In this module the pre-processing of input data set is done by converting all the input data into .csv file. This file will be used for training the input data set.

\section{3) Classification of Data Elements}

In this module the classification of input data set is performed based on some algorithms like svm, k-nn, logistic regression, neural network technique VGG Architecture (Visual Geometry Group) for the detection of sign language. In this application we try to use VGG for finding the meaning based on sign images.

\section{EXPERIMENTAL REPORTS}

We have conducted experiment on several sensitive data using Android Studio IDE for Google's Android operating system, built on JetBrains' IntelliJ IDEA software and designed specifically for developing mobile applications. We took imagenet data set with set of images with collection of alphabets and numbers. Finally we developed an application which can able to show the performance of our proposed application by taking a sample data set with more than 4000 characters under 24 distinct classes which can be formed with all 26 english letters, words and sentences in Indian Sign Language (ISL). In this current application we try to develop the text to sign language translator successfully.

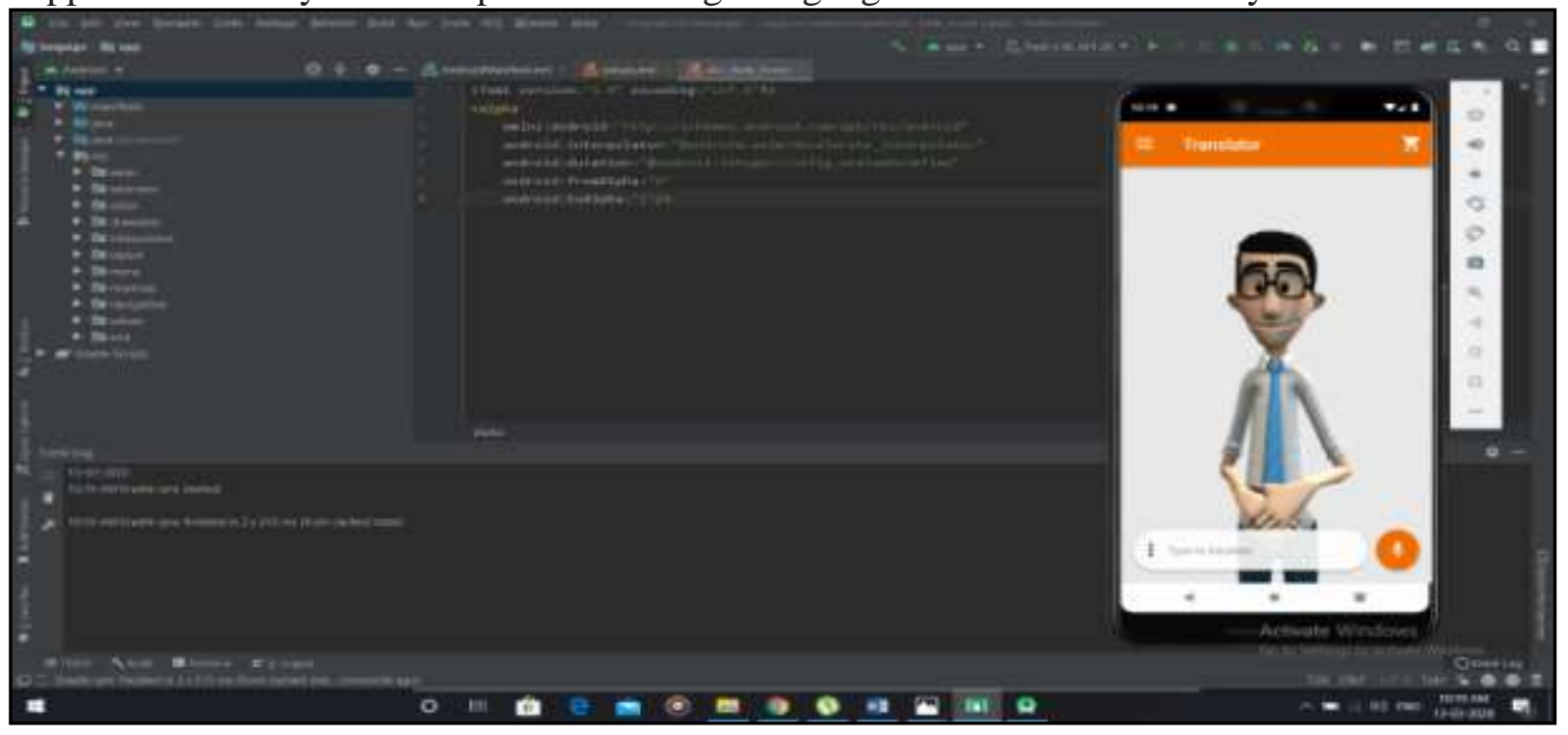

From the above figure, we can able to enter the text in that input message box either through voice based or manual method. Then based on the appropriate input the sign image is formed as the resultant output. 


\section{CONCLUSION}

In this paper, we for the first time developed a mobile application for sign language translator using ML model that will be ready to classify the varied hand gestures used for fingerspelling in sign language. During this user-independent model, classification machine learning algorithms are trained to employ a set of image data and testing is completed on a totally different set of knowledge. In this proposed thesis we try to use a very clear and depth images for obtaining accurate result than compared to previous methods in the literature. Various machine learning algorithms are applied on the datasets, including Convolutional Neural Network (CNN). An effort is formed to extend the accuracy of the CNN model by pre-training it on the Imagenet dataset. By conducting various experiments on our proposed framework, we finally came to a conclusion that our proposed approach is robust and easy to implement text to sign language translator by using mobile application. By using this proposed application we can able to give an option for blind and deaf person with a facility to communicate one with another in an easy manner.

\section{REFERENCES}

[1] Reyes, I. 2005 “Comunicar a través del silencio :las posibilidades de la lengua de signos.

[2] Herrero-Blanco, A., Salazar-Garcia, V. 2005 "Non-verbal predicability and copula support rule in Spanish Sign Language". In: de Groot, Casper / Hengeveld, Kees (eds): Morphosyntactic.

[3] Annelies Braffort,2008 “ TRAITEMENT AUTOMATIQUE DE LA LANGUE DES SIGNES.

[4] SignWriting: um sistema de escrita para linguas de sinais. EFETA - Publicação Pastoral do Surdo, ano 5, n 32. Set/out. 1998

[5] Amaral, M. A., Coutinho, A., \& Martins, M. R. (1994). Para Uma Gramática da Língua Gestual Portuguesa. Lisboa: Caminho.

[6] Finding relevant image content for mobile sign language recognition. Retrieved June 17, 2005, from http://www.techinfo.rwth-aachen.de/Veroeffentlichungen/V001_2001.pdf

[7] Baltazar, A.B. (2010). Dicionário da Língua Gestual Portuguesa. Porto: Porto Editora. Akyol, S., \& Alvarado, P. (2001).

[8] Augustine $\mathrm{Su}$ Project. (n.d.). Retrieved October 1, 2002, from http://www.csdl.tamu.edu/ su/asl

[9] Bowden, R., Zisserman, A., Kadir, T., \& Brady, M. (2003, April). Vision based interpretation of natural sign languages. Paper presented at the Third International Conference on Computer Vision Systems, Graz, Austria.

[10]Furst, J. (2000). Database design for American Sign Language. Paper presented at the 15th International Conference on Computers and their Applications, New Orleans, LA.

\section{ABOUT THE AUTHORS}

P. PERINDHA VALLI is currently pursuing her final year B.Tech in Computer Science and Engineering at Nadimpalli Satyanarayana Raju Institute of Technology (NSRIT), Sontyam, Pendurthi- Anandapuram Highway, Visakhapatnam, India-531173.Her area of interests includes Data Mining and Machine Learning. 
K. CHANDINI is currently pursuing her final year B.Tech in Computer Science and Engineering at Nadimpalli Satyanarayana Raju Institute of Technology (NSRIT), Sontyam, Pendurthi- Anandapuram Highway, Visakhapatnam, India-531173.Her area of interests includes Java.

D. LAABITHA is currently pursuing her final year B.Tech in Computer Science and Engineering at Nadimpalli Satyanarayana Raju Institute of Technology (NSRIT), Sontyam, Pendurthi- Anandapuram Highway, Visakhapatnam, India-531173.Her area of interests includes $\mathrm{C}++$.

G. DURGA BHAVANI SHANKAR is currently pursuing his final year B.Tech in Computer Science and Engineering at Nadimpalli Satyanarayana Raju Institute of Technology (NSRIT), Sontyam, Pendurthi- Anandapuram Highway, Visakhapatnam, India-531173. His area of interests includes Web Technologies and Mobile Applications.

J. SANTOSHI KUMARI is currently working as an Assistant Professor in the Department of Computer Science and Engineering at Nadimpalli Satyanarayana Raju Institute of Technology (NSRIT), Sontyam, Pendurthi- Anandapuram Highway, Visakhapatnam, India531173. She has more than 10 years of teaching experience in various engineering colleges.Her research interests includes Web Technologies ,Data Mining and Machine Learning. 